\title{
Some further applications of KKM theorem in topological semilattices
}

Nguyen The Vinh ${ }^{\mathrm{a}, *}$

${ }^{a}$ Department of Mathematical Analysis, University of Transport and Communications, Hanoi, Vietnam.

This paper is dedicated to Professor Ljubomir Ćirić

Communicated by Professor V. Berinde

\begin{abstract}
In this paper, we obtain some further applications of KKM theorem in setting of topological semilattices such as Ky Fan-Kakutani type fixed point theorem, Sion-Neumann type set-valued minimax theorem, set-valued vector optimization problems.(C)2012 NGA. All rights reserved.
\end{abstract}

Keywords: generalized Ky Fan minimax inequality, set-valued mapping, topological semilattices, $C_{\Delta}$-quasiconvex, upper (lower) $C$-continuous, fixed point, Nash equilibrium.

2010 MSC: Primary 47H10; Secondary 47H04.

\section{Introduction}

In 1961, Ky Fan proved the following famous result:

Theorem 1.1. Let $C$ be a nonempty subset of a Hausdorff topological vector space $X$ and let $T: C \rightarrow 2^{X}$ be such that

1. T is a KKM map, i.e,

$$
\operatorname{conv}\left\{x_{1}, x_{2}, \ldots, x_{n}\right\} \subset \cup_{i=1}^{n} T\left(x_{i}\right)
$$

for every finite subset $\left\{x_{1}, x_{2}, \ldots, x_{n}\right\} \subset C$;

2. $T(x)$ is closed for all $x \in C$;

3. $T\left(x_{0}\right)$ is compact for some $x_{0} \in C$.

\footnotetext{
${ }^{*}$ Corresponding author

Email address: thevinhbn@gmail.com (Nguyen The Vinh)
} 
Then $\bigcap_{x \in C} T(x) \neq \emptyset$.

This important result includes several fundamental mathematical problems, like, Ky Fan minimax inequality, optimization, variational inequality problems and fixed point theorems (see [2]).

In 1996, Horvath and Llinares Ciscar [6] proved topological semilattices version of KKM theorem and gave some applications. Since then, KKM theory is continued in topological semilattices with some papers of Luo [10, 11], Vinh [17, 17, 18].

In this paper, we will continue to study some further applications of KKM theorem in some aspects as Sion-Neumann type set-valued minimax theorem, set-valued vector optimization problems.

The paper is organized as follows. After introduction and preliminaries, in section 3 we prove that Browder-Fan theorem is equivalent to KKM theorem. Section 4 is devoted to a set-valued form of Ky Fan minimax inequality and a set-valued form of Sion-Neumann type minimax theorem. In section 5 we prove an existence result of Pareto equilibria of constrained multiobjective games. The last section is concerned with a Kakutani-Ky Fan type fixed point theorem in topological semilattices with uniform structure.

\section{Preliminaries}

Definition 2.1. ([6]) A partially ordered set $(X, \leqslant)$ is called a sup-semilattice if any two elements $x, y$ of $X$ have a least upper bound, denoted by $\sup \{x, y\}$. The partially ordered set $(X, \leqslant)$ is a topological semilattice if $\mathrm{X}$ is a sup-semilattice equipped with a topology such that the mapping

$$
\begin{aligned}
X \times X & \rightarrow X \\
(x, y) & \mapsto \sup \{x, y\}
\end{aligned}
$$

is continuous.

We have given the definition of a sup-semilattice, we could obviously also consider inf-semilattices. When no confusion can arise we will simply use the word semilattice. It is also evident that each nonempty finite set $A$ of $X$ will have a least upper bound, denoted by $\sup A$.

In a partially ordered set $(X, \leq)$, two arbitrary elements $x$ and $x^{\prime}$ do not have to be comparable but, in the case where $x \leq x^{\prime}$, the set

$$
\left[x, x^{\prime}\right]=\left\{y \in X: x \leq y \leq x^{\prime}\right\}
$$

is called an order interval or simply, an interval. Now assume that $(X, \leq)$ is a semilattice and $A$ is a nonempty finite subset; then the set

$$
\Delta(A)=\bigcup_{a \in A}[a, \sup A]
$$

is well defined and it has the following properties:

1. $A \subseteq \Delta(A)$;

2. if $A \subset A^{\prime}$, then $\Delta(A) \subseteq \Delta\left(A^{\prime}\right)$.

We say that a subset $E \subseteq X$ is $\Delta$-convex if for any nonempty finite subset $A \subseteq E$ we have $\Delta(A) \subseteq E$.

Example 2.2. We consider $\mathbb{R}^{2}$ with usual order defined by

$$
(a, b),(c, d) \in \mathbb{R}^{2},(a, b) \leq(c, d) \Leftrightarrow a \leq c ; b \leq d .
$$

Clearly, $\left(\mathbb{R}^{2}, \leq\right)$ is a topological semilattice.

1. The set

$$
X=\{(x, 1): 0 \leq x \leq 1\} \cup\{(1, y): 0 \leq y \leq 1\}
$$

is $\Delta$-convex but not convex in the usual sense. 
2. The set

$$
X=\{(x, y): 0 \leq x \leq 1 ; y=1-x\}
$$

is convex in the usual sense but not $\Delta$-convex.

Definition 2.3. Let $X$ be a topological semilattice or a $\Delta$-convex subset of a topological semilattice, $Y$ be a topological vector space, $C \subset Y$ be a closed, pointed and convex cone with int $C \neq \emptyset$. A mapping $F: X \rightarrow 2^{Y} \backslash\{\emptyset\}$ is said to be a

1. type I $C_{\Delta}$-quasiconvex mapping if, for any pair $x_{1}, x_{2} \in X$ and for any $x \in \Delta\left(\left\{x_{1}, x_{2}\right\}\right)$, we have either

$$
F(x) \subset F\left(x_{1}\right)-C
$$

or

$$
F(x) \subset F\left(x_{2}\right)-C ;
$$

2. type II $C_{\Delta}$-quasiconvex mapping if, for any pair $x_{1}, x_{2} \in X$ and for any $x \in \Delta\left(\left\{x_{1}, x_{2}\right\}\right)$, we have either

$$
F\left(x_{1}\right) \subset F(x)+C
$$

or

$$
F\left(x_{2}\right) \subset F(x)+C .
$$

We use $\in$ instead of $\subset$ when $F$ is single-valued.

Example 2.4. Let $X=[0,1] \times[0,1]$. We set $x^{1} \leq x^{2}$ denoting that $x^{2} \in x^{1}+\mathbb{R}_{+}^{2}, \forall x^{1}, x^{2} \in X$, where $\mathbb{R}_{+}^{2}=\left\{\left(y_{1}, y_{2}\right) \in \mathbb{R}^{2}: y_{1} \geq 0, y_{2} \geq 0\right\}$. It is obvious that $(X, \leq)$ is a topological semilattice, in which

$$
x^{1} \vee x^{2}=\left(\max \left(x_{1}^{1}, x_{1}^{2}\right), \max \left(x_{2}^{1}, x_{2}^{2}\right)\right), \forall x^{i}=\left(x_{1}^{i}, x_{2}^{i}\right) \in X, i=1,2 .
$$

Let $F, G: X \rightarrow \mathbb{R}$ and $C=-\mathbb{R}_{+}$such that

$$
\begin{aligned}
& F(x)=\left[\left(1-x_{1}\right)\left(1-x_{2}\right),+\infty\right), \forall x=\left(x_{1}, x_{2}\right) \in X . \\
& G(x)=\left(-\infty,\left(1-x_{1}\right)\left(1-x_{2}\right)\right], \quad \forall x=\left(x_{1}, x_{2}\right) \in X .
\end{aligned}
$$

Then $F$ is type II $C_{\Delta^{-}}$-quasiconvex mapping and it is not type I $C_{\Delta^{-}}$-quasiconvex, $G$ is type I $C_{\Delta^{-}}$ quasiconvex mapping and it is not type II $C_{\Delta}$-quasiconvex.

Remark 2.5. If $Y=\mathbb{R}=(-\infty,+\infty)$ and $C=[0,+\infty)$, and $F=\varphi$ is a real function, then the $C$ - $\Delta$ quasiconvexity of $\varphi$ is equivalent to the $\Delta$-quasiconvexity of $\varphi$ (see [10]).

Definition 2.6. ([8], Definition 2.2) Let $X$ be a topological space, $Y$ a topological vector space with a cone $C$. Given a subset $D \subset X$, we consider a multi-valued mapping $F: D \rightarrow 2^{Y}$. The domain of $F$ is defined to be the set $\operatorname{dom} F=\{x \in D: F(x) \neq \emptyset\}$.

1. $F$ is said to be upper (lower) $C$-continuous at $\bar{x} \in d o m F$ if for any neighborhood $V$ of the origin in $Y$ there is a neighborhood $U$ of $\bar{x}$ such that

$$
F(x) \subset F(\bar{x})+V+C \quad(F(\bar{x}) \subset F(x)+V-C, \text { respectively })
$$

holds for all $x \in d o m F \cap U$.

2. If $F$ is upper $C$-continuous and lower $C$-continuous at $\bar{x}$ simultaneously, we say that it is $C$-continuous at $\bar{x}$; and $F$ is upper (respectively, lower) $C$-continuous on $D$ if it is upper (respectively, lower) $C$ continuous at every point of $D$.

3. If $F$ is single-valued, then the upper $C$-continuity and the lower $C$-continuity of $F$ at $\bar{x}$ coincide and we say that $F$ is $C$-continuous at $\bar{x}$. 
Remark 2.7. If $Y=\mathbb{R}$ and $C=\mathbb{R}_{+}=\{x \in \mathbb{R}: x \geq 0\}$ (or $C=\mathbb{R}_{-}=\{x \in \mathbb{R}: x \leq 0\}$ ) and $F$ is $C$-continuous at $\bar{x}$, then $F$ is lower semicontinuous (upper semicontinuous, respectively) at $\bar{x}$ in the usual sense.

Definition 2.8. (Luc [9]) Let $Z$ be a real topological vector space, $C \subset Z$ be a pointed closed convex cone with $\operatorname{int} C \neq \emptyset$, and $A$ be a nonempty subset of $Z$.

1. For $z_{1}, z_{2} \in Z$, denote $z_{1} \leq z_{2}$ if and only if $z_{2}-z_{1} \in C$, and $z_{1}<z_{2}$ if and only if $z_{2}-z_{1} \in \operatorname{int} C$.

2. A point $\bar{z} \in A$ is said to be a vector minimal point (respectively, weakly vector minimal point) of $A$ if for any $z \in A, z-\bar{z} \notin-C \backslash\{0\}$ (respectively, $z-\bar{z} \notin-\operatorname{int} C$ ). Moreover, the set of vector minimal points (respectively, weakly vector minimal points) of $A$ is denoted by $\min _{C}(A)$ (respectively, wimin $(A)$ ).

Lemma 2.9. (Luc [9]) Let $A$ be a nonempty compact subset of a real topological vector $Z$ and $C \subset Z$ be a closed convex cone with $C \neq Z$. Then $\min _{C}(A) \neq \emptyset$.

Definition 2.10. Let $X, Y$ be two topological spaces; $F: X \rightarrow 2^{Y}$ is said to have open lower sections if $F^{-1}(y)=\{x \in X: y \in F(x)\}$ is open for any $y \in Y$.

\section{The equivalence of KKM theorem with Browder-Fan fixed point theorem}

Let us recall two fundamental results of the KKM theory in topological semilattices.

Theorem 3.1. (Horvath and Ciscar [6]) Let $X$ be a topological semilattice with path-connected intervals, $C \subset X$ a nonempty subset of $X$, and $T: C \rightarrow 2^{X}$ be such that:

(1) $T$ has closed values;

(2) $T$ is a KKM mapping;

(3) There exists $x_{0} \in C$ such that the set $T\left(x_{0}\right)$ is compact.

Then we have the set $\cap_{x \in C} T(x)$ is not empty.

Theorem 3.2. (Luo [10]) Let $X$ be a topological semilattice with path-connected intervals and $T: X \rightarrow 2^{X}$ be such that:

(1) For each $x \in X$, the set $T(x)$ is not empty and $\Delta$-convex;

(2) For each $y \in X$, the set $T^{-1}(y)$ is open;

(3) There exists $x_{0} \in C$ such that the set $X \backslash T^{-1}\left(x_{0}\right)$ is compact.

Then there exists $x^{*} \in X$ such that $x^{*} \in T\left(x^{*}\right)$.

To prove the equivalence of these theorems we need some auxiliary results. In what follows, we denote by $\langle B\rangle$ the family of all finite subsets of $B$.

Let $\mathcal{C}$ be the family of all convex subsets of a semilattice $X$ and $A$ is an arbitrary subset of $X$. We set $C O_{\Delta}(A)=\cap\{E \in \mathcal{C}: A \subseteq E\}$.

One can see without difficulty that a subset $E$ of $X$ is $\Delta$-convex if and only if $C O_{\Delta}(E)=E$. The proof of Lemma 2.1 in [14] can be modified accordingly to obtain its version in semilattices as follows:

Lemma 3.3. Let $X$ be a semilattice and $E$ be a nonempty subset of $X$. Then

(1) $C O_{\Delta}(E)$ is a $\Delta$-convex subset of $X$;

(2) $C O_{\Delta}(E)$ is the smallest $\Delta$-convex of $X$ containing $E$;

(3) $C O_{\Delta}(E)=\cup\left\{C O_{\Delta}(A): A \in\langle E\rangle\right\}$.

Proof. (1) Let $A \in\left\langle C O_{\Delta}(E)\right\rangle$. Let $D$ be any $\Delta$-convex subset of $X$ containing $E$. Then $A \subset C O_{\Delta}(E) \subset D$, so $A \in\langle D\rangle$ and hence $\Delta(A) \subset D$. Thus

$$
\Delta(A) \subset \cap\{D: D \text { is a } \Delta \text {-convex subset of } X \text { containing } E\}=C O_{\Delta}(E) .
$$


Therefore $C O_{\Delta}(E)$ is $\Delta$-convex.

(2) It is clear from the definition of $C O_{\Delta}(E)$ and (1).

(3) Let $M=\cup\left\{C O_{\Delta}(A): A \in\langle E\rangle\right\}$. By (1), $M \subset C O_{\Delta}(E)$. On the other hand, it is clear that $E \subset M$. Thus to complete the proof, it suffices to show that $M$ is $\Delta$-convex. Indeed, let $B=\left\{x_{1}, x_{2}, \ldots, x_{n}\right\} \in\langle M\rangle$ be given. Then for each $i=1,2, \ldots, n$, there exists $A_{i} \in\langle E\rangle$ with $x_{i} \in \Delta\left(A_{i}\right)$. Let $A=\cup_{i=1}^{n} A_{i}$, then $A \in\langle E\rangle$ and $B \subset \cup_{i=1}^{n} \Delta\left(A_{i}\right)$. Since $\Delta(A)$ is $\Delta$-convex, $\Delta(B) \subset \Delta(A) \subset M$. Hence $M$ is $\Delta$-convex.

Lemma 3.4. Let $X$ be a topological space and $Y$ be a semilattice. Suppose the mapping $\phi: X \rightarrow 2^{Y} \backslash\{\emptyset\}$ is such that for each $y \in Y, \phi^{-1}(y)$ is open in $X$. Define $\psi: X \rightarrow 2^{Y} \backslash\{\emptyset\}$ by $\psi(x)=C O_{\Delta}(\phi(x))$ for each $x \in X$. Then for each $y \in Y, \psi^{-1}(y)$ is open in $X$.

Proof. Let $y \in Y$ be given. By Lemma 3.1, if $x \in \psi^{-1}(y)$, then

$$
y \in \psi(x)=C O_{\Delta}(\phi(x))=\cup\{\Delta(A): A \in\langle\phi(x)\rangle\} .
$$

Let $A=\left\{a_{1}, a_{2}, \ldots, a_{n}\right\} \in\langle\phi(x)\rangle$ be such that $y \in \Delta(A)$. Then $x \in \cap_{i=1}^{n} \phi^{-1}\left(a_{i}\right)$ which is an open neighbourhood of $x$. Let $U=\cap_{i=1}^{n} \phi^{-1}\left(a_{i}\right)$, then for each $z \in U, a_{i} \in \phi(z)$ for each $i=1,2, \ldots, n$ so that $y \in \Delta(A) \subset C O_{\Delta}(\phi(z))=\psi(z)$. Hence $z \in \psi^{-1}(y)$ for each $z \in U$ and hence $x \in U \subset \psi^{-1}(y)$. Therefore $\psi^{-1}(y)$ is open in $X$.

Now, we are in a position to state the first new result of this paper.

Theorem 3.5. Theorems 3.1 and 3.2 are equivalent.

Proof. Theorem $3.1 \Longrightarrow$ Theorem 3.2 . Let us assume that the conditions of Theorem 3.2 hold. We define $G: X \rightarrow 2^{X}$ by $G(y)=X \backslash T^{-1}(y)$ for each $y \in X$. We have

$$
\bigcap_{y \in X} G(y)=X \backslash \bigcup_{y \in X} T^{-1}(y)=\emptyset
$$

Therefore, $G$ is not a KKM mapping. Hence, there exists $A=\left\{x_{1}, x_{2}, \ldots, x_{n}\right\} \subset X$ such that $\Delta(A) \not \subset$ $\cup_{x \in A} G(x)$. We infer that there exists $x^{*} \in \Delta(A)$ such that $x^{*} \notin G\left(x_{i}\right)$ for all $i=1,2, \ldots, n$. Thus $x^{*} \in T^{-1}\left(x_{i}\right)$ for all $i=1,2, \ldots, n$. It follows that $x_{i} \in T\left(x^{*}\right)$ for all $i=1,2, \ldots, n$. Then $x^{*} \in \Delta(A) \subset T\left(x^{*}\right)$.

Theorem $3.2 \Longrightarrow$ Theorem 3.1: We assume that the conditions of Theorem 3.1 hold. For a contradiction, asumme that, $\cap_{x \in C} T(x)=\emptyset$. Then we can define a set valued mapping $\phi: X \rightarrow 2^{X}$ by $\phi(x)=\{y \in C: x \notin T(y)\}$. Clearly $\phi(x)$ is a nonempty subset of $X$ for each $x \in X$. It follows that for each $y \in X, \phi^{-1}(y)=X \backslash T(y)$ is open in $X$. Let $\psi: X \rightarrow 2^{X}$ be the set-valued mapping defined by $\psi(x)=C O_{\Delta} \phi(x)$ for each $x \in X$. Thus for each $x \in C, \psi(x)$ is a nonempty $\Delta$-convex subset of $X$ and by Lemma 3.4 $\psi^{-1}(y)$ is open for each $y \in X$. Finally, $X \backslash \psi^{-1}\left(x_{0}\right) \subset X \backslash \phi^{-1}\left(x_{0}\right)=T\left(x_{0}\right)$ is compact. Hence by Theorem 3.2 there exists a point $x^{*} \in X$ such that

$$
x^{*} \in \psi\left(x^{*}\right)=C O_{\Delta} \phi\left(x^{*}\right)=\cup\left\{\Delta(A): A \in\left\langle\phi\left(x^{*}\right)\right\rangle\right\} .
$$

This implies that there exists $A=\left\{x_{1}, x_{2}, \ldots, x_{n}\right\} \in\left\langle\phi\left(x^{*}\right)\right\rangle$ such that $x^{*} \in \Delta(A)$. Then $x^{*} \in \phi^{-1}\left(x_{i}\right)=$ $X \backslash T\left(x_{i}\right)$ for $i=1,2, \ldots, n$. This means that $x^{*} \notin T\left(x_{i}\right)$ for $i=1,2, \ldots, n$, i.e, $x^{*} \notin \cup_{i=1}^{n} T\left(x_{i}\right)$, which contradicts the hypothesis (2) of Theorem 3.1. Hence $\cap_{x \in C} T(x) \neq \emptyset$.

\section{Ky Fan inequality and Sion-Neumann minimax theorem for set-valued mappings}

We shall denote by $\sup A(\operatorname{resp} . \inf A)$, where $A \subset Y$, the set of all efficient points of the set $\bar{A}$ (the closure of $A$ ) with respect to $C$ (resp. with respect to $-C$ ), i.e.,

$$
\begin{aligned}
\sup A & =\{a \in \bar{A}:(a+C) \cap \bar{A}=\{a\}\} ; \\
\inf A & =\{a \in \bar{A}:(a-C) \cap \bar{A}=\{a\}\} .
\end{aligned}
$$


Recall that $A$ is bounded with respect to $C$, if the set $(a+C) \cap A$ is bounded for every $a \in A$. A classical lemma of R. Phelps [13, which we shall use in the sequel, states that if $A$ is bounded with respect to $C$ (resp. with respect to $-C)$, then $\sup A \neq \emptyset($ resp. $\inf A \neq \emptyset)$ and

$$
A \subset \sup A-C(\text { resp. } A \subset \inf A+C) .
$$

We shall say that a set-valued mapping $F: X \rightarrow 2^{Y}$, where $X$ is a topological space, is bounded with respect to $C$, if for every $x \in X$ and every $y \in F(x)$ the set $(y+C) \cap F(x)$ is bounded.

We have the following result (see [17, Theorem 3.5] for more general case).

Theorem 4.1. Let $K$ be a nonempty compact $\Delta$-convex subset of a semilattice $X$ with path-connected intervals, $Y$ a topological vector space, $C$ a closed convex pointed cone with intC $\neq \emptyset$ and $F: K \times K \rightarrow 2^{Y}$ a set-valued mapping. Assume that

1. For each $x \in K, F(x, x) \subset-C$;

2. For each $y \in K, F(., y)$ is lower $C$-continuous;

3. For each $x \in K, F(x,$.$) is type I I-C_{\Delta}$-quasiconvex.

Then the solution set

$$
S=\{x \in K: F(x, y) \subset-C, \text { for all } y \in K\}
$$

is a nonempty compact subset of $K$.

Proof. We define $T: K \rightarrow 2^{K}$ by

$$
T(y)=\{x \in K: F(x, y) \subset-C\}, \text { for each } y \in K .
$$

We show that $T(y)$ is closed for each $y \in K$. Taking $\bar{x} \in \overline{T(y)}$, the closure of $T(y)$, we shall deduce that $\bar{x} \in T(y)$. By (2), the lower $C$-continuity of $F(., y)$ implies that for any neighborhood $V$ of the origin in $Y$ there is a neighborhood $U(\bar{x})$ of $\bar{x}$ such that

$$
F(\bar{x}, y) \subset F(x, y)+V-C, \quad \text { for all } x \in U(\bar{x}) .
$$

Let $\left\{x_{\alpha}\right\}$ be any net in $T(y)$ converging to $\bar{x}$, hence there exists $\beta$ such that $x_{\alpha} \in U(\bar{x}), \forall \alpha \geq \beta$ and then

$$
F(\bar{x}, y) \subset F\left(x_{\alpha}, y\right)+V-C, \forall \alpha \geq \beta
$$

and so

$$
F(\bar{x}, y) \subset F\left(x_{\alpha}, y_{i}\right)+V-C \subset-C+V-C \subset-C+V \text { for all } V .
$$

Since $C$ is closed, the last inclusion shows $F(\bar{x}, y) \subset-C$. Therefore, $\bar{x} \in T(y)$ and $T(y)$ is closed.

We shall show that for each $x \in K, P(x)=\{y \in K: F(x, y) \not \subset-C\}$ is $\Delta$-convex. Suppose that there exists an $x^{\prime} \in X$ such that $P\left(x^{\prime}\right)$ is not $\Delta$-convex; then there exist $y^{1}, y^{2} \in P\left(x^{\prime}\right)$ such that $\Delta\left(\left\{y^{1}, y^{2}\right\}\right) \not \subset P\left(x^{\prime}\right)$, i.e., there exists a $z \in \Delta\left(\left\{y^{1}, y^{2}\right\}\right)$ and $z \notin P\left(x^{\prime}\right)$; hence $F\left(x^{\prime}, z\right) \subset-C$. By (3), we have either

$$
F\left(x^{\prime}, y^{1}\right) \subset F\left(x^{\prime}, z\right)-C
$$

or

$$
F\left(x^{\prime}, y^{2}\right) \subset F\left(x^{\prime}, z\right)-C .
$$

Consequently, we have either

$$
F\left(x^{\prime}, y^{1}\right) \subset F\left(x^{\prime}, z\right)-C \subset-C-C \subset-C
$$

or

$$
F\left(x^{\prime}, y^{2}\right) \subset F\left(x^{\prime}, z\right)-C \subset-C-C \subset-C,
$$


which is a contradiction. Therefore, for any $x \in X, P(x)$ is $\Delta$-convex.

Finally, we prove that $T$ is a KKM mapping. Suppose on the contrary that $T$ is not KKM. Then there exists $A=\left\{y_{1}, y_{2}, \ldots, y_{n}\right\} \subset K$ such that

$$
\Delta(A) \not \subset \bigcup_{i=1}^{n} T\left(y_{i}\right) .
$$

Thus there exists $z \in \Delta(A)$ such that $z \notin \bigcup_{i=1}^{n} T\left(y_{i}\right)$. Hence $z \notin T\left(y_{i}\right)$ for all $i=1,2, \ldots, n$. It follows that $y_{i} \in P(z)$ for all $i=1,2, \ldots, n$. Since $P(z)$ is $\Delta$-convex, we have $z \in \Delta(A) \subset P(z)$, i.e., $F(z, z) \not \subset-C$, which contradicts the hypothesis (1). Then $T$ is a KKM mapping. By Theorem 3.1, we infer that

$$
\bigcap_{y \in K} T(y) \neq \emptyset
$$

and the solution set $S=\{x \in K: F(x, y) \subset-C$, for all $y \in K\}$ is a nonempty compact subset of $K$.

Theorem 4.2. Suppose that $X, Y$ are compact topological semilattices with path-connected intervals, $C$ is a closed convex pointed cone with intC $\neq \emptyset$ in a topological vector space and $F, G: X \times Y \rightarrow 2^{E}$ are two set-valued mappings such that the set $\cup_{y \in Y} \sup \cup_{x \in X} F(x, y)$ is bounded with respect to $-C$ and the set $\cup_{x \in X} \inf \cup_{y \in Y} G(x, y)$ is bounded with respect to $C$. Suppose that $F$ and $G$ satisfy the following conditions:

1. $F(x, y)-G(x, y) \subset-C$ for every $x \in X, y \in Y$;

2. $G(x,$.$) is C$ - $\Delta$-quasiconcave on $Y$ for every $x \in X$ and $F(., y)$ is $-C$ - $\Delta$-quasiconcave on $X$ for every $y \in Y$;

3. $G(., y)$ is lower $-C$-continuous for every $y \in Y$ and $F(x,$.$) is lower C$-continuous for every $x \in X$.

Then there exist two points

$$
z_{1} \in \sup \cup_{x \in X} \inf \cup_{y \in Y} G(x, y)
$$

and

$$
z_{2} \in \inf \cup_{y \in K} \sup \cup_{x \in X} F(x, y)
$$

such that $z_{1}-z_{2} \in C$.

Proof. Define the mapping $H: X \times Y \times X \times Y \rightarrow 2^{E}$ by

$$
H(\hat{x}, \hat{y}, x, y)=F(x, \hat{y})-G(\hat{x}, y) .
$$

Applying Theorem 4.1 for $H$ we obtain that there exist $x_{0}, y_{0}$ such that

$$
H\left(x_{0}, y_{0}, x, y\right) \subset-C, \quad \forall x \in X, \forall y \in Y,
$$

whence

$$
\sup \cup_{x \in X} F\left(x, y_{0}\right)-\inf \cup_{y \in Y} G\left(x_{0}, y\right) \subset-C .
$$

Using Phelps lemma stated at the beginning of this section, we have

$$
\sup \cup_{x \in X} F\left(x, y_{0}\right) \subset \inf \cup_{y \in Y} \sup \cup_{x \in X} F(x, y)+C
$$

and

$$
\inf \cup_{y \in Y} G\left(x_{0}, y\right) \subset \sup \cup_{x \in X} \inf \cup_{y \in Y} G(x, y)-C .
$$

Therefore, by (4.1) there exist

$$
z_{1} \in \sup \cup_{x \in X} \inf \cup_{y \in Y} G(x, y), c_{1} \in C
$$


and

$$
z_{2} \in \inf \cup_{y \in K} \sup \cup_{x \in X} F(x, y), c_{2} \in C
$$

such that

$$
z_{2}+c_{2}-\left(z_{1}-c_{1}\right) \in-C
$$

which implies

$$
z_{1}-z_{2} \in C_{1}+c_{1}+c_{2} \subset C
$$

Remark 4.3. Theorem 4.1 is a set-valued version of Ky Fan minimax inequality, while Theorem 4.2 is a set-valued form of Sion-Neumann type minimax theorem in topological semilattices.

\section{The existence of (weak) Pareto equilibria}

The following theorem, the proof of which is contained in the proof of Theorem 3 of Horvath and Llinares Ciscar in [6], will be the basic tool for our purpose.

Theorem 5.1. Let $X$ be a compact topological space, $Y$ be a topological semilattice with path-connected intervals and $T: X \rightarrow 2^{Y}$ have nonempty $\Delta$-convex values and open lower sections. Then there is a continuous selection $f: X \rightarrow Y$ of $T$ such that $f=g \circ h$ where $g: \Delta_{n} \rightarrow Y$ and $h: X \rightarrow \Delta_{n}$ are continuous mappings and $n$ is some positive integer.

Lemma 5.2. Let $I$ be an index set and for each $i \in I$, let $X_{i}$ be a nonempty, compact and $\Delta$-convex subset of a topological semilattice with path-connected intervals and $X=\prod_{i \in I} X_{i}$. For each $i \in I$, let $T_{i}: X \rightarrow 2^{X_{i}}$ be a set-valued mapping such that

1. $T_{i}$ has nonempty $\Delta$-convex values;

2. $T_{i}$ has open lower sections.

Then there exists a point $x \in X$ such that $x \in T(x):=\prod_{i \in I} T_{i}(x)$; that $i s, x_{i} \in T_{i}(x)$ for each $i \in I$, where $x_{i}=\pi_{i}(x)$ is the projection of $x$ onto $X_{i}$ for each $i \in I$.

Proof. By Theorem 5.1, for each $i \in I$, there exists continuous mappings $g_{i}: \Delta_{n_{i}} \rightarrow X_{i}$ and $h_{i}: X \rightarrow \Delta_{n_{i}}$ such that $f_{i}=g_{i} \circ h_{i}$ is a continuous selection of $T_{i}$, where $n_{i}$ is some positive integer. Now let $S=\prod_{i \in I} \Delta_{n_{i}}$. For each $i \in I$, let $E_{i}$ be the linear hull of the set $\left\{e_{0}, e_{1}, \ldots, e_{n_{i}}\right\}$, then $E_{i}$ is a locally convex topological vector space as it is finite dimensional and $\Delta_{n_{i}}$ is a compact convex subset of $E_{i}$. Let $E=\prod_{i \in I} E_{i}$, then $E$ is also a locally convex topological vector space and $S$ is also a compact convex subset of $E$.

Now define continuous mappings $g: S \rightarrow X$ and $h: X \rightarrow S$ by

$$
g(t)=\prod_{i \in I} g_{i}\left(\pi_{i}(t)\right), \forall t \in S \text { and } h(x)=\prod_{i \in I} h_{i}(x), \forall x \in X,
$$

where $\pi_{i}: S \rightarrow \Delta_{n_{i}}$ is the projection of $S$ on $\Delta_{n_{i}}$ for each $i \in I$. By Tychonoff fixed point theorem [15], the continuous mapping $h \circ g: S \rightarrow S$ has a fixed point $t \in S$, i.e., $t=h \circ g(t)$. Let $\bar{x}=g(t)$, then we have

$$
\begin{aligned}
\bar{x} & =g \circ h(\bar{x})=g\left(\prod_{i \in I} h_{i}(\bar{x})\right) \\
& =\prod_{i \in I} g_{i}\left(\pi_{i}\left(\prod_{i \in I} h_{i}(\bar{x})\right)\right)=\prod_{i \in I} g_{i} \circ h_{i}(\bar{x}) .
\end{aligned}
$$

It follows that $\bar{x}_{i}=g_{i} \circ h_{i}(\bar{x}) \in T_{i}(\bar{x})$ for each $i \in I$. This completes the proof.

From Lemma 5.2, we have the following fixed component theorem in topological semilattices. 
Theorem 5.3 ([18]). Let $\left\{X_{i}\right\}_{i \in I}$ be a family of compact $\Delta$-convex sets each in a topological semilattice with path-connected intervals, $X=\prod_{i \in I} X_{i}$, and $\left\{T_{i}: X \rightarrow 2^{X_{i}}\right\}_{i \in I}$ a family of mappings satisfying the following conditions:

1. each $T_{i}$ has $\Delta$-convex values;

2. each $T_{i}$ has open lower sections.

3. for each $x \in X$, there exists $i \in I$ such that $T_{i}(x) \neq \emptyset$.

Then there exists $x=\left(x_{i}\right)_{i \in I} \in X$ and $i \in I$ such that $x_{i} \in T_{i}(x)$.

It is easy to see that Theorem 5.3 is equivalent to the following maximal element theorem for a family of mappings.

Theorem 5.4. Let $\left\{X_{i}\right\}_{i \in I}$ be a family of compact $\Delta$-convex sets each in a topological semilattice with path-connected intervals, $X=\prod_{i \in I} X_{i}$, and $\left\{T_{i}: X \rightarrow 2^{X_{i}}\right\}_{i \in I}$ a family of maps satisfying the following conditions:

1. each $T_{i}$ has $\Delta$-convex values;

2. each $T_{i}$ has open lower sections.

3. for each $x=\left(x_{i}\right)_{i \in I} \in X$ and $i \in I, x_{i} \notin T_{i}(x)$.

Then there exists $\bar{x} \in X$ such that $T_{i}(\bar{x})=\emptyset$ for all $i \in I$.

The above theorem will be used in the main result of this section.

Let $\left(X_{i}, \leqslant_{i}\right), i \in I$, be a family of topological semilattices, and let $X$ and $X_{-i}$ be the product spaces with the product topology, i.e.,

$$
X:=\prod_{i \in I} X_{i}, \quad X_{-i}:=\prod_{j \in I \backslash\{i\}} X_{j},
$$

For $x, x^{\prime} \in X:=\prod_{i \in I} X_{i}$, define $x \leq x^{\prime}$ if and only if $x_{i} \leq_{i} x_{i}^{\prime}$, then $(X, \leqslant)$ is a topological semilattice with $\left[\sup \left\{x, x^{\prime}\right\}\right]_{i}=\sup \left\{x_{i}, x_{i}^{\prime}\right\}$ for each $i \in I$ (see [6]). For any $x \in X, x=\left(x_{-i}, x_{i}\right)$, where $x_{i} \in X_{i}, x_{-i} \in X_{-i}$.

Let $Y$ be a Hausdorff topological vector space. For each $i \in I$, let $A_{i}: X \rightarrow 2^{X_{i}}$ be the $i$ th constraint correspondence and $F_{i}: X \rightarrow 2^{Y}$ the $i$ th pay-off mapping. The following result is Theorem 4.1 in [18].

Theorem 5.5. Let $I$ be any index set and for each $i \in I, X_{i}$ be a nonempty compact $\Delta$-convex subset of a topological semilattice with path-connected intervals,

$$
X:=\prod_{i \in I} X_{i}, \quad X_{-i}:=\prod_{j \in I \backslash\{i\}} X_{j} .
$$

For each $i \in I$, let $Y_{i}$ be a locally convex topological vector space and $A_{i}: X \rightarrow 2^{X_{i}}, F_{i}: X \rightarrow 2^{Y_{i}}, C_{i} a$ closed, pointed and convex cone in $Y_{i}$ with int $C_{i} \neq \emptyset$. Assume that

1. $\forall i \in I, A_{i}$ has open lower sections and nonempty $\Delta$-convex values;

2. $\forall i \in I$, the set $B_{i}=\left\{x \in X: x_{i} \in A_{i}(x)\right\}$ is closed;

3. $\forall i \in I, F_{i}$ is upper $C_{i}$-continuous with closed values;

4. $\forall i \in I, F_{i}\left(x_{-i}, u_{i}\right)$ is lower $-C_{i}$-continuous in $x_{-i}$;

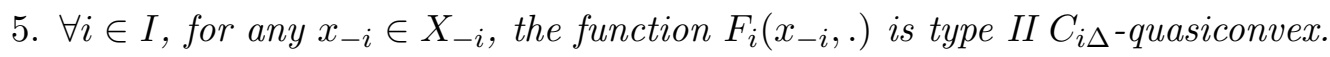

Then there exists $x^{*} \in X$ such that for each $i \in I$,

$$
x_{i}^{*} \in A_{i}\left(x^{*}\right), \quad F_{i}\left(x_{-i}^{*}, u_{i}\right) \subset F_{i}\left(x_{-i}^{*}, x_{i}^{*}\right)+C_{i}, \quad \forall u_{i} \in A_{i}\left(x^{*}\right) .
$$


Let $I$ be any (finite or infinite) index set and for each $i \in I, X_{i}$ be topological semilattices. We still use the following notations $X, X_{-i}$ as in Theorem 5.5. For each $x \in X, x_{i}$ and $x_{-i}$ denote the projection of $x$ on $X_{i}$ and $X_{-i}$ respectively. Write $x=\left(x_{-i}, x_{i}\right)$.

Let $I$ be any set of players. Each player $i \in I$ has a strategy set $X_{i}$, a constrained correspondence $A_{i}$ : $X \rightarrow 2^{X_{i}}$, a payoff $F_{i}: X \times X_{i} \rightarrow 2^{Y_{i}}$, where $Y_{i}$ is a Hausdorff topological vector space, $C_{i}$ is a pointed closed convex cone in $Y_{i}$ with $\operatorname{int} C_{i} \neq \emptyset$ and $C_{i} \neq Y_{i}$. A generalized constrained multiobjective game (GCMOG) $\Gamma=\left(X_{i}, A_{i}, F_{i}, C_{i}\right)_{i \in I}$ is a family of ordered quadruples $\left(X_{i}, A_{i}, F_{i}, C_{i}\right)$. A point $x^{*}=\left(x_{-i}^{*}, x_{i}^{*}\right) \in X$ is said to be a Pareto (resp., weak Pareto) equilibrium point of $\Gamma$ if for each $i \in I$, there exists a point $z_{i}^{*} \in F\left(x_{-i}^{*}, x_{i}^{*}\right)$ such that

$$
\begin{gathered}
x_{i}^{*} \in A_{i}\left(x^{*}\right), \quad z_{i}-z_{i}^{*} \notin-C_{i} \backslash\{0\}, \quad \forall z_{i} \in F_{i}\left(x_{-i}^{*}, u_{i}\right), u_{i} \in A_{i}\left(x^{*}\right) \\
\left(\text { resp. }, x_{i}^{*} \in A_{i}\left(x^{*}\right), \quad z_{i}-z_{i}^{*} \notin-\operatorname{int} C_{i}, \quad \forall z_{i} \in F_{i}\left(x_{-i}^{*}, u_{i}\right), u_{i} \in A_{i}\left(x^{*}\right)\right)
\end{gathered}
$$

Since $-\operatorname{int} C_{i} \subset-C_{i} \backslash\{0\}$, it is easy to see that each Pareto equilibrium point of the GCMOG must be a weak Pareto equilibrium point of the GCMOG.

Theorem 5.6. Let $I$ be any index set and for each $i \in I, X_{i}$ be a nonempty compact $\Delta$-convex subset of a topological semilattice with path-connected intervals, $Y_{i}$ be a locally convex topological vector space, $C_{i}$ be a closed, pointed and convex cone in $Y_{i}$ with int $C_{i} \neq \emptyset$ and $C_{i} \neq Y_{i}$. Let $\Gamma=\left(X_{i}, A_{i}, F_{i}, C_{i}\right)$ be a generalized constrained multiobjective game. For each $i \in I$, let $A_{i}: X \rightarrow 2^{X_{i}}, F_{i}: X \rightarrow 2^{Y_{i}}$ satisfying the following conditions:

1. $\forall i \in I, A_{i}$ has open lower sections and nonempty $\Delta$-convex values;

2. $\forall i \in I$, the set $B_{i}=\left\{x \in X: x_{i} \in A_{i}(x)\right\}$ is closed;

3. $\forall i \in I, F_{i}$ is upper $C_{i}$-continuous with compact values;

4. $\forall i \in I, F_{i}\left(x_{-i}, u_{i}\right)$ is lower $-C_{i}$-continuous in $x_{-i}$;

5. $\forall i \in I$, for any $x_{-i} \in X_{-i}$, the function $F_{i}\left(x_{-i},.\right)$ is type II $C_{i \Delta}$-quasiconvex.

Then there exists $x^{*} \in X$ such that for each $i \in I$, there exists a point $z_{i}^{*} \in F\left(x^{*}\right)$ satisfying

$$
x_{i}^{*} \in A_{i}\left(x^{*}\right), \quad z_{i}-z_{i}^{*} \notin-C_{i} \backslash\{0\}, \quad \forall z_{i} \in F_{i}\left(x_{-i}^{*}, u_{i}\right), u_{i} \in A_{i}\left(x^{*}\right)
$$

i.e., $x^{*} \in X$ is a Pareto equilibrium point of the GCMOG and so $x^{*} \in X$ is also a weak Pareto equilibrium point of the GCMOG.

Proof. First, we prove that there exists $x^{*}=\left(x_{-i}^{*}, x_{i}^{*}\right) \in \prod_{i \in I} X_{i}$ such that for each $i \in I$,

$$
x_{i}^{*} \in A_{i}\left(x^{*}\right), \quad F_{i}\left(x_{-i}^{*}, x_{i}^{*}\right) \cap \min _{C_{i}} F_{i}\left(x_{-i}^{*}, A_{i}\left(x^{*}\right)\right) \neq \emptyset .
$$

If it is false, then for each $x \in \prod_{i \in I} X_{i}$, there exists $i \in I$ such that either

$$
x_{i} \notin A_{i}(x)
$$

or

$$
F_{i}\left(x_{-i}, x_{i}\right) \cap \min _{C_{i}} F_{i}\left(x_{-i}, A_{i}(x)\right)=\emptyset .
$$

But, by Theorem 5.5. there exists $x^{*}=\left(x_{-i}^{*}, x_{i}^{*}\right) \in \prod_{i \in I} X_{i}$ such that for each $i \in I$,

$$
x_{i}^{*} \in A_{i}\left(x^{*}\right) \text { and } F_{i}\left(x_{-i}^{*}, u_{i}\right) \subset F_{i}\left(x_{-i}^{*}, x_{i}^{*}\right)+C_{i}, \quad \forall u_{i} \in A_{i}\left(x^{*}\right) .
$$

Hence we have

$$
F_{i}\left(x_{-i}^{*}, x_{i}^{*}\right) \cap \min _{C_{i}} F_{i}\left(x_{-i}^{*}, A_{i}\left(x^{*}\right)\right)=\emptyset .
$$


By the condition $(3), F_{i}\left(x_{-i}^{*}, x_{i}^{*}\right)$ is compact in $Y_{i}$, it follows from Lemma $2.2, \min _{C_{i}} F_{i}\left(x_{-i}^{*}, x_{i}^{*}\right) \neq \emptyset$. Let $z_{i}^{0} \in \min _{C_{i}} F_{i}\left(x_{-i}^{*}, x_{i}^{*}\right) \subset F_{i}\left(x_{-i}^{*}, x_{i}^{*}\right)$. It follows from t5.3 that

$$
z_{i}^{0} \notin \min _{C_{i}} F_{i}\left(x_{-i}^{*}, A_{i}\left(x^{*}\right)\right) .
$$

Hence, there exist $u_{i}^{*} \in A_{i}\left(x_{-i}^{*}\right)$ and $z_{i}^{*} \in F_{i}\left(x_{-i}^{*}, u_{i}^{*}\right)$ such that

$$
z_{i}^{0} \in z_{i}^{*}+C_{i} \backslash\{0\} .
$$

By (5.5), there exists $z_{i} \in F_{i}\left(x_{-i}^{*}, x_{i}^{*}\right)$ such that

$$
z_{i}^{*} \in z_{i}+C_{i}
$$

By (5.4) and (5.5), we have

$$
z_{i}^{0}-z_{i}=z_{i}^{0}-z_{i}^{*}+z_{i}^{*}-z_{i} \in C_{i} \backslash\{0\}+C_{i}=C_{i} \backslash\{0\} .
$$

which contradicts the fact that $z_{i}^{0} \in \min _{C_{i}} F_{i}\left(x_{-i}^{*}, x_{i}^{*}\right)$. Therefore 5.1 is true. It follows from Definition 2.8 and (5.1) that there exists $x^{*}=\left(x_{-i}^{*}, x_{i}^{*}\right) \in X$ such that for each $i \in I$, there exists $z_{i}^{*} \in F_{i}\left(x_{-i}^{*}, x_{i}^{*}\right)$ satisfying

$$
x_{i}^{*} \in A_{i}\left(x^{*}\right), \quad z_{i}-z_{i}^{*} \notin-C_{i} \backslash\{0\}, \quad \forall z_{i} \in F_{i}\left(x_{-i}^{*}, u_{i}\right), u_{i} \in A_{i}\left(x^{*}\right),
$$

i.e., $x^{*} \in X$ is a Pareto equilibrium point of the GCMOG and so $x^{*} \in X$ is also a weak Pareto equilibrium point of the GCMOG.

\section{Ky Fan-Kakutani type fixed point theorem in topological semilattices}

This section is concerned with a Kakutani-Ky Fan type fixed point theorem in topological semilattices with uniform structure.

Definition 6.1. (Kelly [7]) A uniformity for a set $X$ is a non-void family $\mathcal{U}$ of subsets of $X \times X$ (called entourages) such that

1. each member of $\mathcal{U}$ contains the diagonal $\Omega=\{(x, x) \in X\}$,

2. if $U \in \mathcal{U}$, then $U^{-1} \in \mathcal{U}$, where $U^{-1}=\{(y, x) \in X \times X:(x, y \in U)\}$,

3. if $U \in \mathcal{U}$, then $V \circ V \subset U$ for some $V \in \mathcal{U}$, where

$$
V \circ V=\{(x, z): \exists y \in X \text { such that }(x, y) \in V,(y, z) \in V\},
$$

4. if $U$ and $V$ are members of $\mathcal{U}$, then $U \cap V \in \mathcal{U}$, and

5. if $U \in \mathcal{U}$ and $U \subset V \subset X \times X$, then $V \in \mathcal{U}$.

The pair $(X, \mathcal{U})$ is called a uniform space. For each $V \in \mathcal{U}$, we define a neighborhood of $x$ as $V[x]:=$ $\{y \in X:(x, y) \in V\}$. An entourages $V$ is called symmetric if $V=V^{-1}$. In this case, we have

$$
y \in V[x] \Leftrightarrow x \in V[y] .
$$

Let

$$
\mathcal{O}=\{G \subset X \text { : for each } x \in G \text { there exists } V \in \mathcal{U} \text { such that } V[x] \subset G\} .
$$

Then $\mathcal{O}$ is a topology on $X$, and it called the topology induced by the uniformity $\mathcal{U}$. Moreover, $(X, \mathcal{O})$ is called a uniform topological space.

The uniform space $(X, \mathcal{U})$ is said to be separated if

$$
\bigcap\{V: V \in \mathcal{U}\}=\Omega
$$

in this case $(X, \mathcal{O})$ becomes a Hausdorff space. 
Definition 6.2. A topological semilattice $X$ is said to be a locally $\Delta$-convex space if $X$ is a uniform topological space with uniformity $\mathcal{U}$ which has an open base $\beta:=\left\{V_{i}: i \in I\right\}$ of symmetric entourages such that for each $V \in \beta$, the set $V[x]$ is a $\Delta$-convex for each $x \in X$.

We shall assume that locally $\Delta$-convex spaces also satisfy the following condition:

Condition $(H):\{x \in X: K \cap V[x] \neq \emptyset\}$ is $\Delta$-convex for any $\Delta$-convex subset $K$ of $X$ and $V \in \beta$ (see, Horvath [5, Definition 2, p. 345]).

Definition 6.3. (Berge [1]) Let $X$ and $Y$ be two Hausdorff topological spaces and $F: X \rightarrow 2^{Y}$ be a setvalued mapping, then $F$ is upper semicontinuous at $x_{0} \in X$ if for each open set $U$ in $Y$ with $U \supset F\left(x_{0}\right)$, there exists an open neighborhood $O\left(x_{0}\right)$ of $x_{0}$ such that $U \supset F(x)$ for any $x \in O\left(x_{0}\right) ; F$ is upper semicontinuous on $X$ if $F$ is upper semicontinuous at every point in $X$.

We need the following result.

Theorem 6.4. (Horvath and Ciscar [6]) Let $X$ be a topological semilattice with path-connected intervals, $C \subset X$ a nonempty subset of $X$, and $T: C \rightarrow 2^{X}$ be such that:

1. T has closed [resp., open] values;

2. $T$ is a KKM mapping, i.e., for each $A \in\langle X\rangle$,

$$
\Delta(A) \subset \bigcup_{x \in A} T(x) .
$$

Then the family $\{T(x): x \in C\}$ has the finite intersection property.

Theorem 6.5. Let $X$ be a separated compact locally $\Delta$-convex space with path-connected intervals satisfying the condition $(H)$ and $T: X \rightarrow 2^{X}$ be an upper semicontinuous set-valued mappings with nonempty closed $\Delta$-convex values. Then $T$ has a fixed point, i.e, there exists $x_{0} \in X$ such that $x_{0} \in T\left(x_{0}\right)$.

Proof. Fix an element $V$ of the base $\beta$, then for each $x \in X, V[x]$ is an open neighborhood of $x$. Since $T(X)$ is compact, there exists an $M=\left\{y_{1}, y_{2}, \ldots, y_{n}\right\} \subset X$ such that $T(X) \subset \cup_{y \in M} V[y]$.

For each $y_{i} \in M$, let $G\left(y_{i}\right):=\left\{x \in X: T(x) \cap \overline{V\left[y_{i}\right]}=\emptyset\right\}$. Since $T$ is upper semicontinuous and $\overline{V\left[y_{i}\right]}$ is closed, by a standard argument, we can prove that each $G\left(y_{i}\right)$ is open. Moreover, since $T(X) \subset \cup_{i=1}^{n} V\left[y_{i}\right]$, we have

$$
\bigcap_{i=1}^{n} G\left(y_{i}\right)=\left\{x \in X: T(x) \cap \bigcup_{i=1}^{n} \overline{V\left[y_{i}\right]}=\emptyset\right\}=\emptyset .
$$

Therefore, by Theorem 6.4, $G: M \rightarrow 2^{X}$ cannot be a KKM map; that is, there exist an $N \in\langle M\rangle$ and an $x_{V} \in \Delta(N)$ such that $x_{V} \notin G(N)=\cup_{y \in N} G(y)$. Hence $T\left(x_{V}\right) \cap \overline{V[y]} \neq \emptyset$ for all $y \in N$, and

$$
N \subset L:=\left\{y \in X: T\left(x_{V}\right) \cap \overline{V[y]} \neq \emptyset\right\} .
$$

Since $T\left(x_{V}\right)$ is $\Delta$-convex set and $X$ satisfies the condition $(H), L$ is $\Delta$-convex. Therefore, $x_{V} \in \Delta(N) \subset L$ and hence $T\left(x_{V}\right) \cap \overline{V\left[x_{V}\right]} \neq \emptyset$.

So, for each basis element $V$, there exist $x_{V}, y_{V} \in X$ such that $y_{V} \in T\left(x_{V}\right)$ and $y_{V} \in \overline{V\left[x_{V}\right]}$. Since $T(X)$ is compact and $\beta$ forms a directed set ordered by inclusion, we may assume that the net $\left\{y_{V}\right\}$ converges to some $x_{0} \in K$. Since $X$ is Hausdorff, $x_{V}$ also converges to $x_{0}$. Since $T$ is upper semicontinuous with closed values, the graph of $T$ is closed in $X \times T(X)$, and hence we have $x_{0} \in T x_{0}$. This completes our proof.

\section{Acknowledgements:}

The author would like to thank Professor Do Hong Tan and the members of the seminar "Fixed point theory, KKM theory and Applications" at Hanoi National University of Education, for many helpful discussions in preparation of this paper. The author thanks the referee for the valuable comments and suggestions. 


\section{References}

[1] C. Berge, Espaces Topologiques, Fonctions Multivoques, Dunnod, Paris, 1959. 6.3

[2] E. Blum and W. Oettli, From optimization and variational inequalities to equilibrium problems, Math. Student 63 (1994), 123-145. 1

[3] P. Deguire and M. Lassonde, Familles sélectantes, Topol. Methods Nonlinear Anal. 5 (1995), 261-269.

[4] K. Fan, A minimax inequality and applications. In, O. Shisha, ed., Inequalities III, Proceedings of the third Symposium on inequalities, Academic Press, New York, 1972.

[5] C. D. Horvath, Contractibility and Generalized Convexity, J. Math. Anal. Appl. 156 (1991), 341-357. 6

[6] C. D. Horvath and J. V. Llinares Ciscar, Maximal elements and fixed points for binary relations on topological ordered spaces, J. Math. Econom. 25 (1996), 291-306. 1, 2.1, 3.1, 5, 5, 6.4

[7] J. L. Kelly, General Topology, Van Nostrand, Princeton, NJ, 1955. 6.1

[8] L.-J. Lin and N. X. Tan, On quasivariational inclusion problems of type I and related problems, J. Glob. Optim. 39 (2007), 393-407. 2.6

[9] D. T. Luc, Theory of Vector Optimization. In, Lecture Notes in Economics and mathematical systems, Vol. 319, Springer-Verlag, Berlin, 1989. 2.8, 2.9

[10] Q. Luo, KKM and Nash equilibria type theorems in topological ordered spaces, J. Math. Anal. Appl. 264 (2001), 262-269. $1,2.5,3.2$

[11] Q. Luo, The applications of the Fan-Browder fixed point theorem in topological ordered spaces, Appl. Math. Lett. 19 (2006), 1265-1271. 1

[12] J. W. Peng and X. M. Yang, On existence of a solution for the system of generalized vector quasi-equilibrium problems with upper semicontinuous set-valued maps, Inter. J. Math. Math. Sciences 15 (2005), 2409-2420.

[13] R. R. Phelps, Convex functions, monotone operators and differentiablity, Lecture Notes in Mathematics, SpringerVerlag, Vol. 1364, 1989. 4

[14] K.-K. Tan, X.-L. Zhang, Fixed point theorems on G-convex spaces and applications, Proc. Nonlinear Func. Anal. Appl. 1 (1996), 1-19. 3

[15] A. Tychonoff, Ein Fixpunktsatz, Math. Ann. 111 (1935), 767-776. 5

[16] N. T. Vinh, Matching theorems, fixed point theorems and minimax inequalities in topological ordered spaces, Acta Math. Vietnam. 30 (2005), 211-224.

[17] N. T. Vinh, Some generalized quasi-Ky Fan inequalities in topological ordered spaces, Vietnam J. Math. 36 (2008), 437-449. 1, 4

[18] N. T. Vinh,Systems of generalized quasi-Ky Fan inequalities and Nash equilibrium points with set-valued maps in topological semilattices, PanAmer. Math. J. 19 (2009), 79-92. 1, $5.3,5$

[19] G. X. Z. Yuan, KKM Theory and Applications in Nonlinear Analysis, Marcel Dekker Inc., New York, 1999. 